\title{
Calculations of radio pulses from High Energy Showers
}

\author{
Jaime Alvarez-Muñiz \\ Bartol Research Institute, University of Delaware, Newark, DE 19711. \\ alvarez@bartol.udel.edu \\ Enrique Zas \\ Departamento de Física de Partículas, \\ Universidad de Santiago de Compostela, E-15706 Santiago, Spain. \\ zas@fpaxp1.usc.es
}

\begin{abstract}
In this article we review the progress made in understanding the main characteristics of coherent Čerenkov radiation induced by high energy showers in dense media. A specific code developed for this purpose is described because it took a significant part in this process. Subsequent approximations developed for the calculation of radio pulses from $\mathrm{EeV}$ showers are reviewed. Emphasis is given to the relation between the shower characteristics and different features of the corresponding radio emission.
\end{abstract}

\section{INTRODUCTION}

It was about 40 years ago that Askar'yan proposed the detection of very high energy particles through the coherent radio emission from all the particles in a high energy shower [1]. Although radio emission from showers had been discussed before, Askar'yan noted that showers would develop an excess of electrons of order $10 \%$ independently of shower energy and that such excess would lead to coherent radiation. Indeed if the emission is coherent the electric field scales as the excess charge and as the shower energy rises, the relative contribution of radio emission increases with respect to other wavelengths (say optical Cerenkov). Since the number of particles in a shower is proportional to shower energy, the power in the coherent signal scales with the square of the primary energy. This fact together with the technical simplicity of detectors makes the technique attractive for detecting very high energy showers. It is remarkable that many detection possibilities addressed in this meeting were already in Askar'yan original work.

Clearly coherence demands that the wavelength of the radiation exceeds the size of the radiating region. As electromagnetic showers develop through successive pair 
production and bremsstrahlung interactions, the particle distributions of showers of a given energy are, to a very rough approximation, similar when described in radiation length units ${ }^{1}$. The radiation length of different materials in units of matter depth basically scales with the inverse of the atomic number, being $37 \mathrm{~g} \mathrm{~cm}^{-2}$ for air and not too different for many other abundant materials that have been proposed for radio experiments such as water, salt and sand. Because of the medium density the scale of the showers can however be completely different. While air showers have sizes in the $\mathrm{km}$ range and coherence effects can be obtained at frequencies of order $10 \mathrm{MHz}$, for showers in ice the scale is a meter and coherence is kept to frequencies of order $10 \mathrm{GHz}$.

Since the power associated to the Cerenkov emission increases with frequency Askar'yan suggested detecting showers in dense medium, as those that could be induced by deeply penetrating particles underground or by cosmic rays on the Moon surface. Attempts were made to detect radio pulses in coincidence with other air shower detectors [2] and, although the results were positive, it became clear that there were many difficulties. Calculations revealed that several additional mechanisms competed with excess charge in the generation of radio pulses, including dipole radiation and transverse currents because of the magnetic field of the Earth, and transition radiation when the shower reached the ground [3]. The interpretation of radio signals was obscured by these and other difficulties and the technique was almost completely abandoned but for a few isolated efforts that have been reviewed in this conference.

In the mid 1980's the efforts to build high energy neutrino telescopes renewed the activity in the radio technique $[4,5]$ as a possible alternative to the projects to detect the Cerenkov light from muons under water or ice [7]. As a result early in the 1990's a Monte Carlo program was developed for the calculation of distant radio signals from TeV electromagnetic showers in ice [8,9]. These led to several efforts to assess the possibility to detect neutrino events with arrays of antennas in Antarctica [10-12]. Later on a series of approximations were made to extend the results of these calculations to both electromagnetic and hadronic showers of energies in the EeV and ZeV ranges [13-16]. In this article we will concentrate on the efforts made towards understanding Čerenkov radio emission in dense media from the excess charge that develops in high energy showers. The recent experimental confirmation of this effect in a SLAC experiment [17], in agreement with expectations, leaves now little doubt about the enormous potential of this technique. We believe that many developments will follow this conference and it is our hope that these calculational efforts will help in the task.

1) Hadronic showers can be regarded as a jet of hadrons along shower axis which generates a chain of electromagnetic subshowers through neutral pion decays and have similar scales. 


\section{ZHS: A SHOWER GENERATOR FOR RADIO EMISSION.}

The calculation of radio pulses from showers is a complex problem in which the emission from all particles in a high energy shower have to be carefully added in a coherent fashion, taking into account the velocity and direction, position and time of each of the particles involved. For a medium such as ice, having a refraction index $n=1.78$ for radio waves, electrons of energy exceeding $107 \mathrm{keV}$ emit Cerenkov radiation. The simulation of the shower cascade from this perspective was tackled by Zas, Halzen and Stanev in [9] with a result that has become known as the ZHS Monte Carlo. Since the largest contribution to the excess charge in a shower is from electrons, only electromagnetic showers were considered. This program has been a reference point for much progress made during the 1990's in radio detection and deserves a brief description. Efforts have been made by other groups to test the code against other shower codes [18].

The longitudinal development of an electromagnetic shower mostly follows from successive pair production and bremsstrahlung interactions with the electric field of atomic nuclei that are the heart of the showering process. For both these processes the effective interaction distance increases as the energy of the interacting photon or electron rises and atomic screening of the atoms becomes progressively more important. In this respect the Landau-Pomeranchuck-Migdal (LPM) effect can be regarded as the higher energy extreme of this situation in which the atomic potential of the individual atoms has to be modified because of collective effects of several nuclei. The lateral structure of the shower is mostly controlled by elastic scattering. Because of the long range of the Coulomb interaction it turns out that most angular deviations arise because of multiple elastic scattering which is treated in this Monte Carlo keeping the first two terms in Molière's expansion [19].

Besides these interactions that are common to all simulation programs, the ZHS code has special features for radio calculations. It is a three dimensional code that takes timing of the particles in great detail. Time delays are measured with respect to a plane perpendicular to shower axis that starts in phase with the primary particle and moves parallel to the axis at speed $c$. Most of such delays are due to angular deviations, to multiple elastic scattering and to the subluminal velocities of the shower particles all of which are taken into account. The program includes electron positron annihilation, Möller, Bhabha and Compton scattering, which only become relevant at low energies but are responsible for the excess charge in the shower. These routines were developed following the EGS4 code [20]. In the first version [8] it did not include the LPM effect but this was soon remedied [9]. It can be run with threshold energies as low as $100 \mathrm{keV}$, but its accuracy is expected to decrease for thresholds below this value because it does not include the photoelectric effect. Finally it is designed to be fast using, when possible, numerical parameterizations instead of actual functions to minimize calculating time.

The results obtained for the longitudinal development of the showers are con- 
sistent with the Greisen parameterization. The lateral distributions are consistent with three dimensional theoretical shower results provided the Molière radius is divided by a factor of about 2 , in agreement with other simulations [21]. The excess charge obtained is of order $20 \%$ depending on particle threshold, it is only slightly dependent on shower depth, see Fig. 1a. The extra track due to electrons is $21 \%$ of the total (the Cerenkov emission is proportional to the tracklengths of the charged particles) as shown in Fig. 1b. Compton scattering of atomic electrons that get accelerated into the shower account for most of the effect $(12 \%)$. It is remarkable that close to half $(14 \%)$ of the excess tracklength is due to particles below $5 \mathrm{MeV}$ $(1 \mathrm{MeV})$. The calculation of coherent signals requires a lower threshold than implied by these numbers because the lower energy particles are expected to interfere more destructively as they get longer delays and larger angular deviations. This is already important because it sets an upper bound to the energy threshold necessary for an accurate calculation.

\section{III ČERENKOV RADIATION}

The detection of radio pulses from air showers is clearly a very different problem from detecting them in a dense medium such as ice. Firstly in ice the coherence
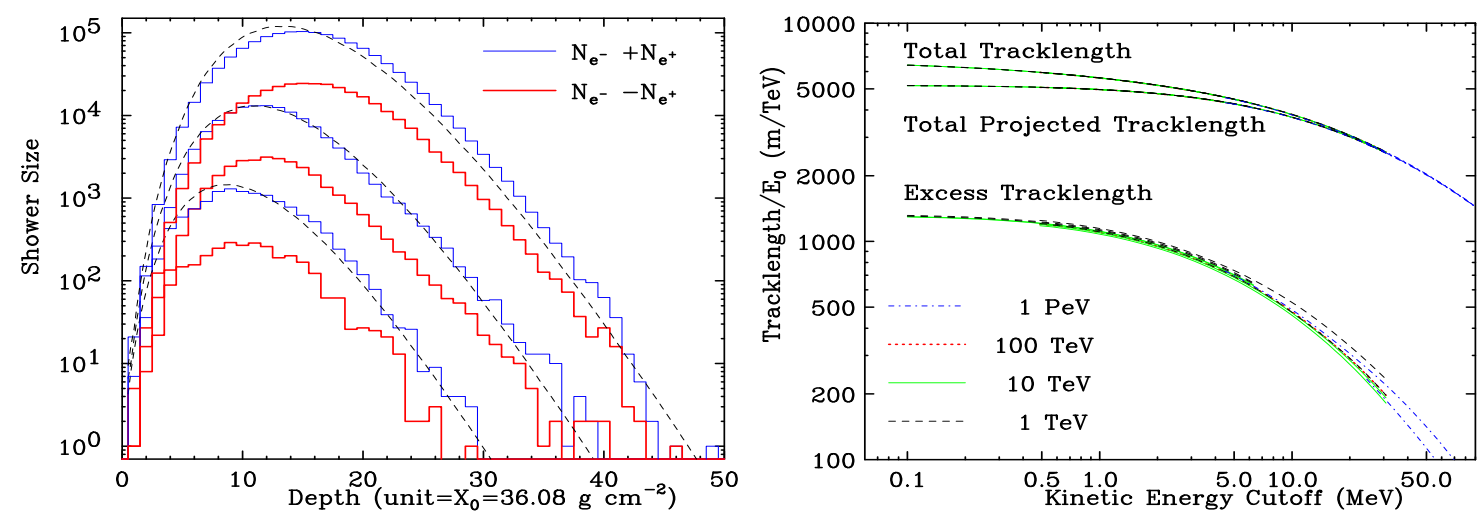

FIGURE 1. a) Left: Depth development of the total number of electrons and positrons and the excess number of electrons for single 1, 10 and $100 \mathrm{TeV}$ electron initiated showers. The Monte Carlo threshold energy is $1 \mathrm{MeV}$. Depth is measured in radiation lengths of ice. The dashed lines are the results in approximation-B of shower theory in the limit of $E_{t h} \rightarrow m_{e}$. b) Right: Shower track lengths for all charged particles in 1, 10, $100 \mathrm{TeV}$ and $1 \mathrm{PeV}$ electron showers as a function of the calculational cutoff $\left(E_{t h}\right)$. The three sets of curves correspond to i) the sum of all track lengths (Total Tracklength), ii) the sum of all track lengths projected onto the shower axis (Total Projected Tracklength) and iii) the difference of electron and positron track lengths projected onto the shower axis (Excess Tracklength). Several showers are plotted for each energy to illustrate the small effect of fluctuations. The tracklengths have been normalized to the shower energy in $\mathrm{TeV}$. 
band extends to the GHz regime. Secondly most shower particles travel very small paths of tens of centimeters, and they are hardly deviated because of the Earth's magnetic field. As a result the excess charge mechanism is expected to dominate the radiation and the problem becomes simpler from the calculation point of view. Moreover there is a substantial difference in the relation between the typical observation distances and the shower dimensions. While for air showers the observation distance is of the some order or even less than the shower dimensions, prospects of detecting radio pulses under the Earth surface or from the Moon are attractive precisely because they can be detected from very large distances compared with the shower dimensions which are of order one meter. For most interesting cases the Fraunhofer approximation turns out to be accurate what is useful for calculational purposes and simplifies both the interpretation and the presentation of results.

When a particle of charge $z$ moves through a medium of refractive index $n$ with velocity $|\vec{v}|=\beta c>c / n$ Cerenkov light is emitted at the Cerenkov angle $\theta_{C}$, verifying $\cos \theta_{C}=(\beta n)^{-1}$, with a power spectrum given by the well known Frank-Tamm result [22] :

$$
\frac{d^{2} W}{d \nu d l}=\left[\frac{4 \pi^{2} \hbar}{c} \alpha\right] z^{2} \nu\left[1-\frac{1}{\beta^{2} n^{2}}\right],
$$

with $\nu$ the frequency, $c$ the speed of light, $d l=c \beta d t$ a small element of particle track length, and $\alpha$ the fine structure constant. This is the standard approximation used for most Čerenkov applications for wavelengths orders of magnitude smaller than the tracks. This expression corresponds to an infinite track and in the case of a finite track there are diffraction effects.

Working with the time Fourier transform of the electric field, $\vec{E}(\omega)$, Maxwell's equations can be solved in the transverse gauge for an arbitrary current [9]:

$$
\vec{E}(\omega, \overrightarrow{\mathrm{x}})=\frac{\mathrm{e} \mu_{\mathrm{r}}}{2 \pi \epsilon_{0} \mathrm{c}^{2}} i \omega \iiint \int \mathrm{dt}^{\prime} \mathrm{d}^{3} \overrightarrow{\mathrm{x}^{\prime}} \mathrm{e}^{i \omega \mathrm{t}^{\prime}+i|\vec{k}|\left|\overrightarrow{\mathrm{x}}-\overrightarrow{\mathrm{x}^{\prime}}\right|} \frac{\overrightarrow{\mathrm{J}_{\perp}}\left(\mathrm{t}^{\prime}, \overrightarrow{\mathrm{x}^{\prime}}\right)}{\left|\overrightarrow{\mathrm{x}}-\overrightarrow{\mathrm{x}^{\prime}}\right|}
$$

where $|\vec{k}|=k=n \omega / \mathrm{c}$ and $\vec{J}_{\perp}$ is a divergenceless component of the current transverse to the observation direction, $\mu_{r}$ is the relative permeability of the medium and $\epsilon_{0}$ the permittivity of the vacuum. Eq. 1 can be obtained from this expression. For a single particle moving uniformly with velocity $\vec{v}$ between times $t_{1}$ and $t_{1}+\delta t$ in the Fraunhofer limit it simplifies to:

$$
\vec{E}(\omega, \overrightarrow{\mathrm{x}})=\frac{e \mu_{\mathrm{r}} i \omega}{2 \pi \epsilon_{0} \mathrm{c}^{2}} \frac{\mathrm{e}^{i k R}}{R} \mathrm{e}^{i(\omega-\vec{k} \cdot \vec{v}) \mathrm{t}_{1}} \vec{v}_{\perp}\left[\frac{\mathrm{e}^{i(\omega-\vec{k} \cdot \vec{v}) \delta \mathrm{t}}-1}{i(\omega-\vec{k} \cdot \vec{v})}\right]
$$

The Fraunhofer limit corresponds to an observation distance $R>>[v \delta t \sin \theta]^{2} / \lambda$. For a characteristic track of one radiation length $(\sim 40 \mathrm{~cm})$ and $\lambda$ of $30 \mathrm{~cm}$ corresponding to $1 \mathrm{GHz}$ frequencies, the condition implies $R>>50 \mathrm{~cm}$ which is well 
satisfied for detection distances of order one $\mathrm{km}$. It should be remarked that the inequality can be always satisfied provided a sufficiently small time interval is chosen. The ZHS program uses this expression. For an infinitesimal track $\delta l$ it becomes:

$$
R \vec{E}(\omega, \overrightarrow{\mathrm{x}})=\frac{e \mu_{\mathrm{r}} i \omega}{2 \pi \epsilon_{0} \mathrm{c}^{2}} \vec{\delta} l_{\perp} \mathrm{e}^{i\left(\omega-\vec{k} \vec{v}_{1}\right) \mathrm{t}_{1}} \mathrm{e}^{i k R}
$$

This expression shows that the electric field amplitude is proportional to the tracklength and that radiation is polarized in the direction of $\vec{l}_{\perp}$, the apparent direction of the track as seen by the observer.

For a high energy shower the calculation of the radio pulse is now a matter of book-keeping. Results of the electric field amplitude in the Čerenkov direction display a characteristic frequency spectrum: a linear rise with frequency until the $\mathrm{GHz}$ region where destructive interference sets in and the spectrum gradually levels off as shown in Fig. 2a. Although in the Čerenkov direction all points of the trajectory of a particle moving at the speed of light along the shower axis are emitting in phase, lateral deviations of the particle trajectories and time delays destroy the coherent interference at sufficiently high frequencies. The effect of the lateral distribution is dominant. The amplitude at a given frequency is proportional to the excess tracklength which in turn accurately scales with shower energy as shown in Fig. $1 b$.
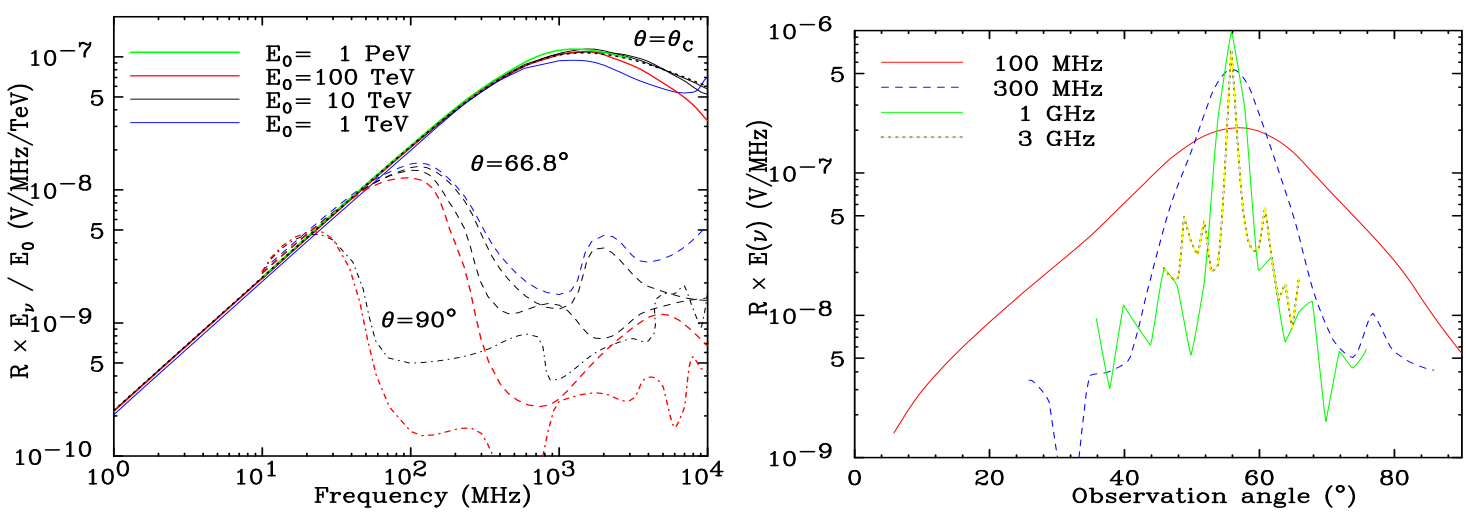

FIGURE 2. a) Left: Frequency spectrum of the electromagnetic pulse. The figure displays 1, 10 and $100 \mathrm{TeV}$ showers for different thresholds. The amplitudes have been divided by the energy of the primary in $\mathrm{TeV}$ and renormalized depending on the threshold. The full curves correspond to observation at the Čerenkov angle $\left(\theta_{C}\right)$ to the shower axis. The dashed (dot-dashed) curve corresponds to observation at $66.8^{\circ}\left(\right.$ at $\left.90^{\circ}\right)$ to the same axis. b) Right: Angular distribution of the electric field generated by a $10 \mathrm{TeV}$ shower $\left(E_{t h}=611 \mathrm{keV}\right)$. The observation angle is the polar angle of the radiation with respect to the shower axis.

The polar diagram of the emitted radiation displays a characteristic peak in the Cerenkov direction of a width which is inversely proportional to the frequency. This is plotted in Fig. 2b and corresponds to a diffraction-like pattern. The width of the diffraction peak about the Čerenkov angle depends on the longitudinal distribution 
of the charge in the shower. As the observation angle gets away from the Cerenkov direction different longitudinal sections of the shower development start to interfere destructively and the amplitude of the electric field drops.

Long tracks in a shower can be subdivided in arbitrary subintervals. Provided the Fraunhofer approximation is valid for the shower as a whole, the algorithm of Eq. 3 for a particle that moves at constant speed displays cancellations between all the end points of the intermediate subintervals of the track. Only the end points of the total track need to be considered in this case what simplifies the computational task enormously. In approximation "a" (default) each electron gives rise to a single track to which Eq. 3 is applied. As particles do suffer some deceleration along the track the average velocity is used. Different criteria for this subdivision have given rise to approximations "b" and "c" [23]. The approximation "a" has been shown to provide sufficiently good results when compared to longer calculations in which each electron track is subdivided into subtracks each of which contributing with Eq. 3 as shown in Fig. 3. Note that approximation "a" is conservative in the sense that it underpredicts the amplitude of the pulse at very high frequencies.

\section{1-D APPROXIMATION}

With the help of scaling it is possible to raise the shower simulation threshold and to generate showers of about $1 \mathrm{PeV}$ but at higher energies the calculations become too lengthy to be handled. The calculation of radioemission from EeV showers is however of great interest because the technique is expected to be most competitive for these energies. Because of the LPM suppression of bremstrahlung and pair production, these showers have very different elongated depth distributions. As a result the angular width of the Cerenkov peaks is expected to be reduced with respect to showers of energy below $1 \mathrm{PeV}$. As the lateral distributions of these showers are not expected to be very different from the lower energy showers, not many effects can be anticipated in the frequency spectrum of the signal in the Čerenkov direction.

The one dimensional approximation has been developed to study the effects of the longitudinal elongation $[13,14]$. In this approximation the particles in the shower are all assumed to be moving at the speed of light and the lateral distribution is neglected. The current density simply becomes [16]:

$$
\overrightarrow{J_{\perp}}\left(\overrightarrow{\mathrm{x}^{\prime}}, \mathrm{t}^{\prime}\right)=Q\left(z^{\prime}\right) \vec{c}_{\perp} \delta^{3}\left(\overrightarrow{\mathrm{x}^{\prime}}-\hat{n}_{z} c t^{\prime}\right)
$$

where the shower develops along the $z$ axis, $\hat{n}_{z}$ is a unit vector in this direction and $Q\left(z^{\prime}\right)$ is the excess charge in the shower. The substitution of this current into Eq. 2 leads to:

$$
\vec{E}(\omega, \overrightarrow{\mathrm{x}})=\frac{e \mu_{\mathrm{r}}}{2 \pi \epsilon_{0} \mathrm{c}^{2}} i \frac{\omega}{c} \sin \theta \hat{n}_{\perp} \int d z^{\prime} Q\left(z^{\prime}\right) \frac{e^{i \frac{\omega}{c} z^{\prime}+i k\left|\overrightarrow{\mathrm{x}}-z^{\prime} \hat{n}_{z}\right|}}{\left|\overrightarrow{\mathrm{x}}-z^{\prime} \hat{n}_{z}\right|}
$$




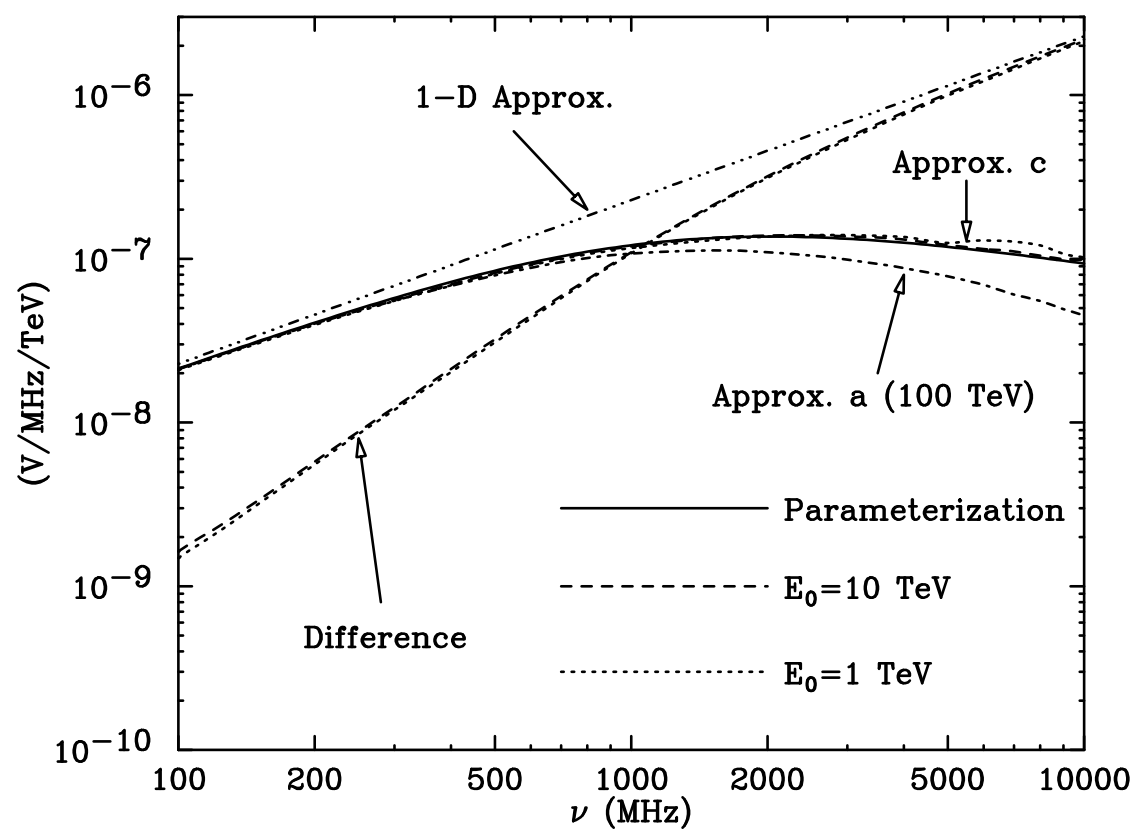

FIGURE 3. Comparison of complete simulation results for the frequency spectrum in the Čerenkov direction for 1 and $10 \mathrm{TeV}$ electromagnetic showers in approximation $c$, for a $100 \mathrm{TeV}$ in the standard approximation $(a)$ and with the 1D-approximation (top curve). The lower curves represent the difference between the $1 \mathrm{D}$ approximation and the full simulation results using in the $c$ approximation. Note that both the spectrum and the difference have the same behavior for different shower energies. All radio pulses scale with shower energy and are normalized to $1 \mathrm{TeV}$.

where $\theta$ is the angle between the shower axis and the direction of observation $\vec{x}$ and $\hat{n}_{\perp}$ is a unitary vector perpendicular to $\overrightarrow{\mathrm{x}}$. It should be stressed that no further approximations are made at this stage.

The phase factor in Eq. 6 can be approximated by $i k\left|\vec{x}-\vec{z}^{\prime}\right| \simeq i k R-i \vec{k} \dot{\vec{z}}^{\prime}$ in the Fraunhofer limit. Here $R=|\overrightarrow{\mathrm{x}}|$ is the distance from the center of the shower to the observation point. The electric field amplitude simply becomes the Fourier transform of the longitudinal charge distribution:

$$
\vec{E}(\omega, \overrightarrow{\mathrm{x}})=\frac{e \mu_{\mathrm{r}}}{2 \pi \epsilon_{0} \mathrm{c}^{2}} i \omega \sin \theta \frac{\mathrm{e}^{i k R}}{R} \hat{n}_{\perp} \int d z^{\prime} Q\left(z^{\prime}\right) \mathrm{e}^{i p z^{\prime}}
$$

where we have introduced for convenience the parameter $p(\theta, \omega)=(1-n \cos \theta) \omega / c$ in Eq. 7. The angular pattern around the Cerenkov direction is precisely the analog of the classical diffraction pattern of an aperture function.

The accuracy of the approximation has been studied comparing it to complete simulations. Excellent agreement is obtained for frequencies below $100 \mathrm{MHz}$. For frequencies progressively increasing above this value, the electric field becomes overestimated in the Cerenkov direction, but the angular distribution of the pulse is otherwise preserved. This is not surprising since the lateral distribution is ignored and no levelling off in the frequency spectrum can be expected. Moreover the dif- 
ference between the complete simulation and the approximation scales with shower energy as shown in Fig. 7. Ad hoc corrections can be implemented to make quantitative predictions in the $\mathrm{GHz}$ regime accounting in an effective way for the ignored effects $[14,16]$. This is analogous to the form factor described in [24]. The approximation gives some insight into the complexity of the spectrum and angular distribution of the pulses relating them to features in shower development.

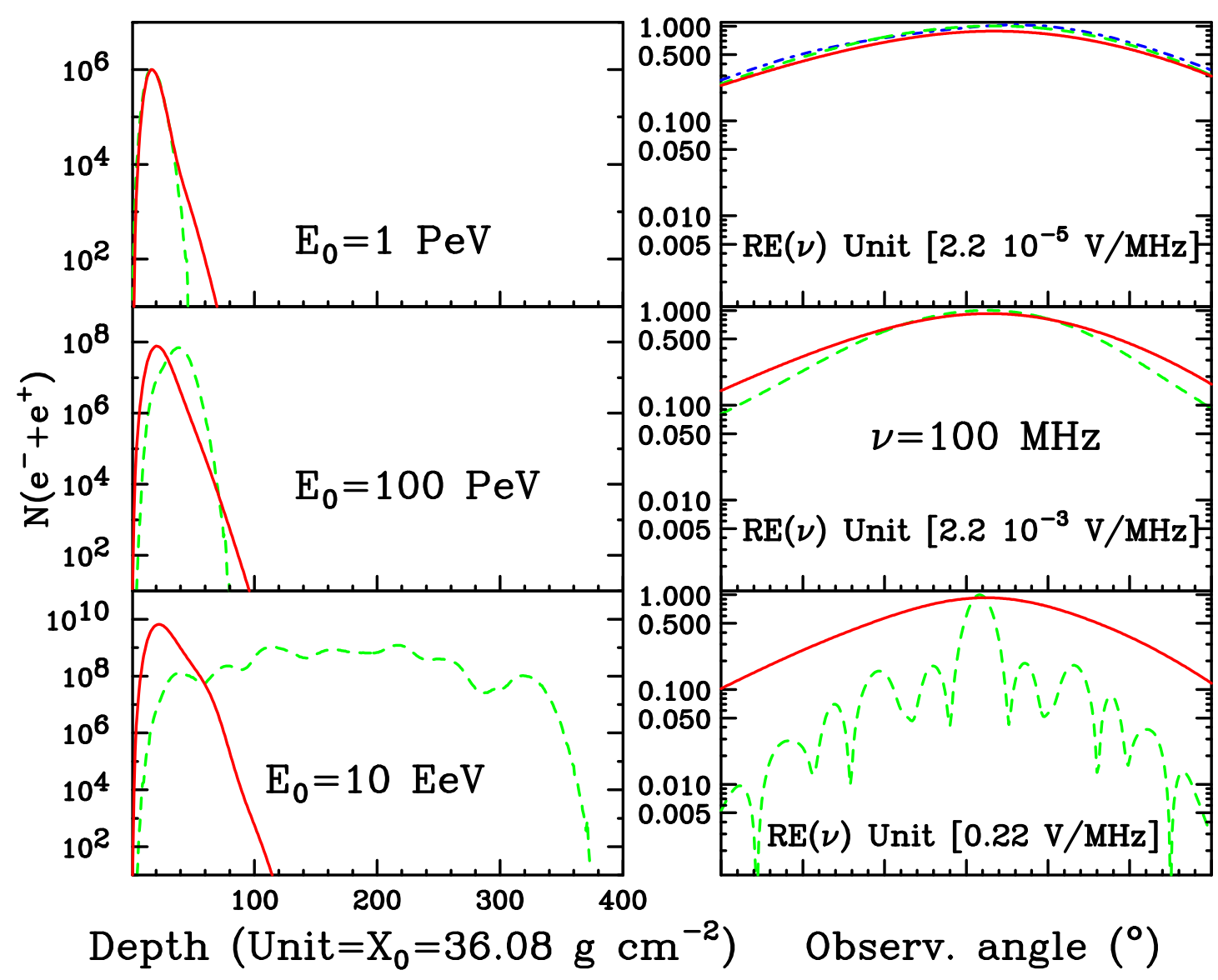

FIGURE 4. Left: Longitudinal development of electromagnetic (solid red lines) and hadronic (dashed lines) showers in ice for different energies. Right: Angular distribution of the electric field amplitude times the distance emitted by the showers shown on the left. Shown is the value $|E(\nu) R|$ where $R$ is the distance to the shower, normalized to its maximum at the Cherenkov angle $\left(\theta_{C}=56^{\circ}\right)$. The units, that is the precise values at maximum are marked in the figure. For the $1 \mathrm{PeV}$ case the electric field amplitude obtained in a complete simulation (dot-dashed line) is also shown to compare with the $1 \mathrm{D}$ approximation.

The development of EeV showers of electromagnetic [13] and hadronic [14] character has been studied using hybrid techniques that combine simulation and parameterizations of low energy showers. The LPM suppression leads to a very large elongation of electromagnetic showers which results in a reduction of the angular width of the diffracted pulses. The elongation has been calculated to scale as $E_{s h}^{1 / 3}$ 
for shower energies satisfying $E_{s h}>20 \mathrm{PeV}$ [13]. For hadronic showers the elongation does not affect shower development significantly until energies in the EeV range. This is because high energy hadronic interactions have large multiplicities and the particles that emerge carry small fractions of the primary energy. Moreover the neutral pions at very high energies interact before decaying into photons, and the transfer of energy to electromagnetic particles is postponed until the pions have energies in the few 10's of PeV. The emerging photons induce ordinary showers. Photons or electrons from very short lived particles are the main source of the occasional shower elongations observed [14]. This is illustrated in Fig. 4.

Eq. 6 can be also used for calculating the radio emission in an approximate fashion to establish Fresnel interference effects. Since this approximation neglects the lateral distribution the results are only expected to be valid for showers which are very elongated because of the LPM effect. For these showers the Fresnel interference effect becomes more important than the lateral distributions. As the observation distance decreases the angular distribution of the pulses becomes wider and the main peak drops. The results show that at the Fresnel distance defined as $R_{F}=\pi n \nu\left(L_{s} \sin \theta / 2\right)^{2} / c\left(L_{s}\right.$ is the shower length) deviations of the electric field in the Cerenkov direction from the Fraunhofer approximation are below $10 \%$.

For neutrino induced showers the two types of showers become relevant. Neutrinos always induce a hadronic character shower through the nucleon fragments. In the case of charged current electron neutrino interactions, the emerging shower has mixed hadronic and electromagnetic character and the relative weights of each component depend on $y$, the fraction of energy transferred to the nuclear fragments. For very high energy neutrinos this leads to an interesting combination of two showers of different lengths because of the relative importance of the LPM effect for both types of showers. The resulting angular distributions of the radio pulse displays a complex pattern of the two corresponding angular widths. It has been shown that it is in principle possible to use the angular information of the radio pulse to extract the corresponding value of $y$ for the interaction [15].

\section{SUMMARY}

We have reviewed the development in calculations of the full diffraction patterns and frequency spectra of hadronic and electromagnetic showers developing in ice. The complex emerging patterns are well understood in terms of the shower properties. We have stressed the dependence on the angular width of the Cerenkov pulse on length and its implication for electromagnetic showers with strong LPM elongations.

\section{ACKNOWLEDGEMENTS}

We thank D. Saltzberg for helpful comments after carefully reading the manuscript and the organizers P. Gorham and D. Saltzberg for providing this 
unique oportunity to give a good impulse to this field. This work was supported in part by the European Science Foundation (Neutrino Astrophysics Network N. 86), by the CICYT (AEN99-0589-C02-02) and by Xunta de Galicia (PGIDT00PXI20615PR). The research activities of J.A-M at Bartol Research Institute are supported by the NASA grant NAG5-7009.

\section{REFERENCES}

1. G.A. Askar'yan, Zh. Eksp. Teor. Fiz 41, 616 (1961) [Soviet Physics JETP 14 441, (1962)]; 48, 988 (1965) [21, 658 (1965)].

2. T. Weekes, in these proceedings and references therein.

3. F.D. Kahn and I. Lerche, Proc. of the Roy. Soc. A 289, 206 (1966).

4. M.A. Markov, I.M. Zheleznykh, Nucl. Instr. and Methods Phys. Res. A248, 242 (1986).

5. Ralston, J.P. and McKay, D.M., Proc. Astrophysics in Antarctica Conference, ed. Mullan, D.J., Pomerantz, M.A. and Stanev, T., (American Institute of Physics, New York, 1989) Vol. 198, p. 241

6. K. Mannheim, Astropart. Phys. 3, 295 (1995).

7. F. Halzen, in these proceedings.

8. F. Halzen, E. Zas, and T. Stanev, Phys. Lett. B257 432 (1991).

9. E. Zas, F. Halzen, and T. Stanev, Phys. Rev. D45, 362 (1992).

10. A.L. Provorov, I.M. Zheleznykh, Astroparticle Physics 4, 55 (1995).

11. G.M. Frichter, J.P. Ralston, D.W. Mc Kay, Phys. Rev. D 53, 1684 (1996).

12. J.V. Jelley, Astropart. Phys. 5, 255 (1996).

13. J. Alvarez-Muñiz and E. Zas, Phys. Lett. B411, 218 (1997).

14. J. Alvarez-Muñiz and E. Zas, Phys. Lett. B434, 396 (1998).

15. J. Alvarez-Muñiz, R.A. Vázquez and E. Zas, Phys. Rev. D 61, 023001 (2000).

16. J. Alvarez-Muñiz, R.A. Vázquez, E. Zas, Phys. Rev. D 61, 023001 (2000).

17. D. Saltzberg in these proceedings, P. Gorham, et. al, e-print archive: hepex/0011001.

18. D. Besson et al., in these proceedings.

19. Molière, G., Z. Naturforsch 3a 78 (1948).

20. Nelson, W.R., Hirayama, H. and Rogers, D.W.O., The EGS4 Code System, SLAC Rep. SLAC-265, UC-32, Stanford Linear Accelerator Center (1985).

21. M. Hillas, private communication.

22. Frank, I. and Tamm, I., Dokl. Akad. Nauk SSSR 14109 (1937).

23. J. Alvarez-Muñiz, G. Parente, and E. Zas, Proc. XXIV Int. Cosmic Ray Conf., Rome (1995), vol 1, p. 1023.

24. R. Buniy, J.P. Ralston, astro-ph/0003408. 